\title{
Colorectal cancer and continence F173-F186
}

USING AN AUDIT OF 400 CASES OF COLORECTAL CANCER TO ESTABLISH GUIDELINES FOR AN OPEN ACCESS COLONOSCOPY SERVICE.

Greenaway JR, Tayyab GN, Cann PA, Idle NL, Corbett WA, Ritchie C. Depts. of Gastroenterology \& Histopathology, Middlesbrough.

400 consecutive cases of colorectal carcinoma representing approximately 2.5 years of clinical activity by 7 general surgeons and 2 gastroenterologists (all endoscopists) serving a population of 300,000 were identified from the histopathology computer. All case notes were analysed using a proforma with emphasis on presenting symptoms in relation to age and tumour site and time intervals along the referral to operation pathway.

$43 \%$ of patients were female. The mean age was 68 years (range: 35 to 100 years). $58 \%$ of lesions were colonic ( $28 \%$ sigmoid, $3 \%$ descending, $8 \%$ transverse $\& 19 \%$ ascending) and $42 \%$ rectal.

$12 \%$ were regarded as "acute" cases with the interval from GP referral to operation being less than 7 days. For the remaining $88 \%$ (352) "non acute" cases the same interval had a mean of 68.4 days. $47 \%$ of these were operated on within 6 weeks and $86.2 \%$ within 12 weeks of GP referral.

$91.2 \%$ of patients were symptomatic with combinations of rectal bleeding, altered bowel habit and colonic pain. $8.8 \%$ presented with anaemia in the absence of any of these symptoms

Applying various models of referral guidelines to the "non acute" patients referred for the investigation of symptoms allowed prediction of a potential tumour detection rate. Independent variation of the presenting symptom(s), age limit for referral and investigation employed were applied to the database. e.g.

Endoscopy to the SPLENIC FLEXURE for rectal bleeding at any age and/or FULL COLONOSCOPY for change in bowel habit aged 45 years or over.

This strategy results in the potential to detect $96 \%$ of all symptomatic "non acute" patients' tumours.

These referral guidelines form the basis of the open access lower G.I. endoscopy service now offered to primary care physicians in Teesside.

\section{STAGING COLORECTAL NEOPLASIA USING COLONOSCOPIC ENDOLUMINAL ULTRASOUND (EUS)}

Norton SA. Durdey P, Roe AM*, Thomas MG.

Department of Surgery, Bristol Royal Infirmary and Southmead Hospital* Bristol.

Curative resection underpins the successful treatment of colorectal neoplasia. The local excision of adenomas and $T_{1}$ carcinomas (with favourable histology) produces results comparable to radical resection. An accurate assessment of the mural extent of disease should facilitate pre-operative staging and help to stratify patients into those requiring radical resection and those suitable for local excision or minimal access surgery.

We have been evaluating the use of colonoscopic EUS in patients with rectosigmoid neoplasia. A GF-UM20 echoendoscope (Olympus), producing 7.5 and $12 \mathrm{MHz}$ images was used to visualise 19 lesions in 16 patients (median age 74, range 39-83). Mural involvement was assigned as $\mathrm{uT}_{0 / 1}-\mathrm{uT}_{4}$ based on a five layered model of the bowel wall as seen at EUS. Histological assessment (based on the TNM classification of neoplastic disease)is available on 18 lesions with a median distance of $8 \mathrm{~cm}$ from the anal margin (range $4-25 \mathrm{~cm}$ ). EUS accurately staged the disease in 16 lesions, overstaged the disease in 1 lesion and could not stage the disease with certainty in 1 lesion. This represents a good statistical correlation using a Kappa inter-observer analysis (kappa $0.8, \mathrm{p}<0.0001$ ).

Our early experience confirms that we are able to stage rectosigmoid neoplasia with EUS and our excellent concordance with histology is very encouraging. Colonoscopic EUS has the potential to be a powerful diagnostic tool in the assessment of left sided colonic neoplasia and will help plan treatment.

\section{CAN DISTAL ADENOMAS PREDICT PROXIMAL} \section{COLON CANCER?}

Jones R, Moran A*

Gloucester Gastroenterology Group, Gloucestershire Royal Hospital, Gloucester GL1 3NN and North Devon District Hospital*, Barnstaple EX31 4JB

Background: Flexible sigmoidoscopy is proposed as a population screening method to reduce colorectal cancer mortality by prevention and early detection. A subsequent colonoscopy is recommended if a significant distal adenoma is found. Proximal colon cancer is increasing in incidence and will only be detected or prevented by this screening strategy if distal neoplasia can predict its occurrence.

Aims: This study aims to determine the prevalence or past history of significant distal adenomas in patients with a recent diagnosis of proximal colon cancer. This will indicate the effect of a flexible sigmoidoscopy screening strategy on proximal colon cancer mortality. Methods: Patients with histological confirmation of proximal colon cancer were identified from a 30-month period (April 1990 to October 1992) using the local histology database (Gloucestershire Royal Hospital) and the regional cancer registry. Details of presentation, past medical history and subsequent colorectal investigations were obtained from the hospital case notes.

Results: Ninety-two patients had colon cancer proximal to the splenic flexure and six of these were excluded from further analysis (three with ulcerative colitis, one with carcinoid, one with familial adenomatous polyposis and one with hereditary non-polyposis colorectal cancer). Of the remaining 86 patients, only four had a significant distal adenoma identified (all were tubulo-villous adenomas), although only $24(28 \%)$ had flexible sigmoidoscopy or colonoscopy and 31 (43\%) had a barium enema (usually preceded by rigid sigmoidoscopy). The distal colon was not investigated in the remaining twenty five patients (29\%).

Conclusions: These results indicate that flexible sigmoidoscopy screening is likely to detect or prevent only a small minority of proximal colon cancers. Prevention or early detection of proximal colon cancers will largely have to depend on other screening strategies such as risk stratification according to family history.
HIGH RESOLUTION MRI : A PROMISING TECHNIQUE FOR PREOPERATIVE STAGING IN RECTAL CANCER G Brown, CJ Richards,AG Radcliffe,PD Carey,M Bourne, GT Williams

Departments of Radiology, Histopathology and Surgery, University Hospital of Wales, Heath Park, Cardiff CF4 4XN

Accurate preoperative assessment of extent of local tumour spread influences patient treatment strategy and prognosis. Staging by digital rectal examination or endorectal ultrasound have limitations. We report a pilot study of the feasibility of high resolution magnetic resonance imaging (HRMRI).

Nine patients with biopsy proven rectal cancer underwent preoperative HRMRI with $3 \mathrm{~mm}$ contiguous image slices through the rectum and pararectal tissues. Following surgery, HRMRI was repeated on each resection specimen, which was then seriaily sectioned at $3 \mathrm{~mm}$ intervals. The extent of the tumour In each slice was mapped histologically.

HRMRI allowed visualisation of all five layers of the recta wall in every case. Correlation of corresponding HRMRI and histopathological slices was initially used to establish criteria for image interpretation. Subsequent comparison of 83 postoperative HRMRI slices with their histological counterparts revealed:

\begin{tabular}{lllllll} 
& \multicolumn{2}{c}{ Histology } & \multicolumn{3}{c}{ T } & \multicolumn{2}{c}{ Stage } \\
MRI T & & T0 & T1 & T2 & T3 & T4 \\
Stage (TNM) & 6 & 3 & 0 & 0 & 0 \\
& T1 & 0 & 11 & 0 & 1 & 0 \\
& T2 & 0 & 1 & 21 & 0 & 0 \\
& T3 & 0 & 1 & 5 & 30 & 0 \\
& T4 & 0 & 0 & 0 & 0 & 4
\end{tabular}

(TNM)

The MRI and histology agreement for stage was $87 \% \quad(k=.81$, $\mathrm{CI}=0.71-0.91$ )

HRMRI is a potentially accurate means of preoperative local staging of rectal cancer that warrants further assessment. 
CRITICAL EVALUATION OF THE ROLE OF ANGIOGENESIS IN COLORECTAL CANCER. C.Roshanlall, G.O'Dowd”, D.Lowe, A.Kinsella, J.Winstanley. University Departments of Surgery and Pathology*; Royal Liverpool Hospital; Liverpool.

It is generally believed that angiogenesis is essential for the growth, maintenance and spread of solid tumours. Tumour angiogenesis, quantified by microvessel density( MVD), has been demonstrated to have a predictive value for certain tumours, notably, breast cancer. Although colorectal cancer is one of the common solid tumours, there are conflicting reports on the role of angiogenesis in colorectal cancer.

We quantified angiogenesis in the invasive tumour and in the corresponding uninvolved mucosa in 100 patients who underwent surgery for primary colorectal adenocarcinoma between 1989-1991 (follow-up period >5yr) and investigated it's relation to Duke's stage and survival . $4 \mu \mathrm{m}$ sections were stained with the monoclonal antibody $\mathrm{QB} / \mathrm{end} / 10$, using the standard $\mathrm{ABC}$ immunohistochemical technique. MVD was measured by two independent observers in i) tumour centre(TC), ii) tumour periphery (TP), iii) tumour free colonic mucosa immediately adjacent to tumour periphery(MA), and iv) normal colon at a distance of at least $2 \mathrm{cms}$ from tumour periphery(N), using two techniques: a) counting in ten equally spaced fields and b) counting in three "hot spots" in each region at $250 \mathrm{X}$ (field area: $0.43 \mathrm{~mm}^{2}$ ).

The results are expressed as mean \pm standard deviation of mean.

$\begin{array}{llllc} & \text { TC } & \text { TP } & \text { MA } & \text { N } \\ \text { Hot spot fields } & 48.9 \pm 24 & 53.4 \pm 20.2 & 68.8 \pm 35.1 & 108.9 \pm 35 \\ \text { Equally spaced fields } & 33 \pm 17 & 37 \pm 14 & 69 \pm 34 & 98 \pm 22\end{array}$

Normal colonic mucosa demonstrated a significantly higher $(p<0.001$, paired $t$ test) MVD compared to TC or TP using either counting technique. Furthermore, there was no significant correlation between MVD in the TC or TP and Duke's stage and survival.

The results of this study suggest that colorectal cancers do not induce a significant angiogenic response and MVD may not have prognostic value in colorectal carcinomas.
SPONTANEOUS APOPTOSIS CORRELATES WITH RESPONSE OF RECTAL CANCER TO COMBINATION CHEMOTHERAPY/ RADIOTHERAPY

A Hale, P Hand, F A Adab, M Deakin, G T Williams, N Scott Department of Biological Sciences, Keele University; Departments of Pathology, Clinical Oncology, and Surgery, North Staffordshire NHS Trust, Stoke-on-Trent.

Pre-treatment biopsies from 21 patients undergoing combination chemotherapy/radiotherapy were assessed for the rate of spontaneous tumour cell apoptosis, mitotic rate, abnormal p53 protein expression and bcl-2 protein expression by immunohistochemistry. A significant positive correlation was found between a high level of spontaneous apoptosis and good response of the tumour to chemo/radiotherapy as determined by the amount of residual carcinoma in the subsequent surgical resection specimen. No correlation was found between tumour response to treatment and mitotic rate, $\mathrm{p} 53$ or bcl-2 protein expression. In particular of 6 pacients where no residual tumour could be found, 3 were p53 protein positive and 2 expressed high levels of bcl-2.

Measuring the rate of apoptosis in pre-treatment biopsies may predict how well a tumour will respond to cytotoxic therapy. No correlation was found with p53 and bcl-2 expression, two genes thought to regulate apoptosis in cancer cells.
The distribution of Kirsten - ras mutations within early colorectal adenocarcinomas

\section{H.J.N. Andreyev, ${ }^{1,2}$ S.A. Sampson, D. Cunningham, ${ }^{1}$} J.V.T.Tilsed ${ }^{1} \&$ P.A. Clarke. ${ }^{2}$

CRC Sections of Medicine, The GI Unit' \& Centre for Cancer Therapeutics, ${ }^{2}$ Dept of Histopathology, The Royal Marsden Hospital \& Institute of Cancer Research, Sutton, Surrey. UK. (Dr. H.J.N. Andreyev is supported by the British Digestive Foundation)

Background: We have previously shown that detection of mutations in codons 12 or 13 of $K i$-ras in whole tissue samples from early colorectal adenocarcinomas do not predict relapse. However, tumours may not be homogeneous for Ki-ras genotype and a few mutated cells may be responsible for subsequent relapse. Therefore, would microdissection of specific areas of colorectal tumours increase the rate of detection of mutations and are there differences between regions within the tumour? Methods: Blocks were retrieved from patients who had undergone apparently curative resection for early colorectal adenocarcinoma but subsequently developed tumour recurrence. Blocks from patients with no relapse after more than 5 years follow up were also used. PCR amplification and direct sequencing to determine $K i$-ras status was used firstly, in whole tissue samples, secondly, in microdissected samples prepared from the $1 \mathrm{~mm}$ leading edge of tumour and thirdly, in microdissected samples from the central tumour core at least $2 \mathrm{~mm}$ away from the leading edge.

Results: Eighteen patients with relapse and 5 long term survivors were identified. Astler-Coller modification of Dukes staging was stage A in 2, B1 in 18 and B2 in 3 patients. A sequence was obtained in all 23 whole tissue samples, in 22 of the leading tumour edges and 20 of the tumour centres. The same genotype was always found in both microdissected samples from the same tumour. In 4 tumours these were mutated. No mutation was found in a microdissected sample which was not detected in the whole tissue sample. In 1 patient, a mutation was detected in the whole tissue sample, but not in the microdissected samples. It is likely that this mutation arose from the epithelium surrounding the tumour rather than from the tumour itself.

Conclusions: These findings suggest that colorectal tumours are homogeneous for Ki-ras genotype and that using PCR amplification followed by direct sequencing of whole tissue samples is accurate, so microdissection of tumours is not necessary. These results also support the hypothesis that the Ki-ras status of a colorectal adenocarcinoma is determined early during it's development.
F180

\section{ALTERED EXPRESSION OF MUC 5AC MUCIN GENE IN} TUBULAR, TUBULO-VILLOUS AND VILLOUS ADENOMAS. PA Sylvester, N Myerscough, MG Thomas, P Durdey, S Biddolph*, BF Warren*, +I Carlstedt, AP Corfield. Depts. of Medicine and Surgery, Bristol Royal Infirmary, Bristol. *Dept. Cellular Pathology, John Radcliffe Hospital, Oxford. and +Dept. Medical and Physiological Chemistry, Lund University, SWEDEN.

Villous adenomas of the rectosigmoid are common and are associated with a baseline recurrence rate. Expression of MUC 5AC transcripts in villous adenomas of the rectum and adjacent histologically normal rectal mucosa has recently been reported. We have extended these observations and studied a potential correlation between mature mucin expression and mRNA levels.

In-situ hybridisation with radioactive mucin oligonucleotide probes on tubular, tubulovillous and villous adenomas as well as normal rectal mucosal specimens was performed. The mature mucin gene product was detected by immunocytochemistry with a novel antiMUC 5AC polyclonal antibody.

No MUC 5AC transcripts were found in the normal rectal specimens. There was trace expression in tubular adenomas $(n=4 / 7)$ and weak expression in both tubulo-villous adenomas $(n=4 / 7)$ and villous adenomas $(n=5 / 9)$.Immunocytochemistry with anti-MUC 5AC antibody showed the absence of supranuclear staining and absent or weak goblet cell staining in control rectum. The tubular(14), tubulovillous (10) and villous(12) groups showed increased MUC5AC staining at both the supranuclear and goblet cell locations. There was a trend to higher expression in the villous adenoma group.

Immunohistochemistry of the mature mucin product supports our in situ hybridization findings and demonstrates the appearance of a noncolonic mucin in adenomas. Immunolocalisation of mature mucins may in the future provide a new, accurate and simple diagnostic marker of recurrence. 
PREVALENCB OF OULPBCTAL CANCER(CRC) IN PATIENIS WITH RECTAL BLBEIING(RB) EAFECT OF AGE

MR THOMPSON, D ARMSTRONG-JAMES, S MOSS, D PRYTHERCH Queen Alexandra Hospital, Portsmouth

\section{Introduction}

On the basis of an Australian study 1 which showed a 1:10 prevalence of CRC in patients wi th RB presenting in General Practice, Goulston 1 advised all RB patients over 40 should have colonoscopy. Is this policy appropriate in England?

\section{Method}

A Portsmouth Postal Survey ${ }^{2}$ of 6,000 patients determined age specific incidence(ASI) of RB in the total population(TP), in patients visiting their GPs(GPP) and in those referred to hospital(HP). Applying

these incidence rates in the total population in

Portsmouth the total number of patients having RB in each decade, was calculated for TP, GPP and HP. The ASI of CRC patients presenting wi th overt RB was calculated from the Wessex CRC Audit.

Results

46,000 patients over 35 have RB in Portsmouth each year, 12,000 visit GPs and 3,500 are referred to hospital. There are 113 RB CRC patients each year in Portsmouth giving CRC prevalence rates of $1: 420,1: 116$ and 1:32 in TP, GPP and HP respectively. Prevalence in GPP varied from $1: 1313$ (35-45yrs) to $1: 41$ over $75 y r s$.

\section{Conclusion}

There does not appear to be as high prevalence rates of CRC in RB in GPP in Portsmouth as in Australid.

\section{References}

1. How important is RB in diagnosis of cancer. Goulston Lancet (1986)

2. Incidence of RB in Portsmouth. Thompson GUT $(37,1995)$
DOES DELAYED GASTRIC EMPTYING IN IDIOPATHIC SLOW TRANSIT CONSTIPATION RETURN TO NORYAL AFTER COLECTONY? David Hemingway, IG Finlay.

Department of Coloproctology, Royal Infirmary of Glasgow, Glasgow

Idiopathic slow transit constipation (ISTC) may be a pan-gastrointestinal motility disorder ince there is evidence of delayed gastric emptying. We studied gastric emptying in patients undergoing colectomy and ileorectal anastomosis (STC IRA) to determine whethor delayed gastric emptying reverts to normal postoperatively.

Patients with ISTC had solid phase gastric emptying

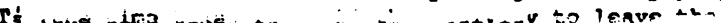

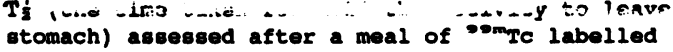
cornflakes before and at one and 12 months after sTC * IRA.

Nine patients had $T$ assessed before and at a mean of one month after surgery. Gastric emptying was abnormally prolonged in all patients preoperatively (mean $\mathrm{T}$ = 127 minutes, range 76-180 minutes, upper ilmit of normal 60 minutes). At one month postoperatively the $\mathrm{Tl}$ was prolonged in all patients (mean $\mathrm{T}$ = 114 minutes, range 100-120 minutes). Of 7 patients at a mean of 14 months postoperatively, the mean $T$ was 64.5 minutes (range 37-115 minutes) and was abnormally prolonged in 3 patients.

This study shows that gastric emptying returns to normal in some, but not all patients who have colectomy for ISTC. This would suggest that some patients have a pan-gastrointestinal motility disorder while in others the abnormality may be confined to colon.
A RETROSPECTIVE STUDY - ILEAL POUCH ANAL ANASTOMOSIS AND COLORECTAL CANCER IN PATIENTS PRESENTING WITH CHRONIC ULCERATIVE COLITIS OR FAMILIAL ADENOMATOUS POL YPOSIS. FUNCTIONAL AND CANCER OUTCOMES. E Radice, R Farouk, H Nelson, JH Pemberton, RR Dozois.

The surgical management of patients with cancer complicating chronic ulcerative colitis (CUC) or familial adenomatous polyposis (FAP) is controversial. Aim: To investigate the long-term outcome of patients with cancer and CUC or FAP undergoing ileal pouch-anal anastomosis (IPAA)

Patients and Methods: of 1615 patients with ileal pouch anal anastomosis from 1981 to 1994 , we identified 85 patients (61 CUC, 24 FAP) with a mean age of 36,8 (range 13-58) years were assessed at a mean of 5 (range 0, 25-14) years after IPAA. Cancer was diagnosed preoperatively in 59 patients. Thirty-six had single rectal cancer and 32 had single colon cancer; multicentricity was found in 15 patients Using the TNM staging classification, 9 patients had carcinoma in situ, 29 had stage 1 cancer, 15 had stage 2,18 had stage 3 and 4 had stage 4.

Results: at a mean follow-up of 5 years, 74 patients $(62,9 \%)$ are alive. Failure to reverse the defunctioning ileostomy occurred in 8 patients (distal disease 5; locally recurrent disease 2; pouch radiotherapy injury 2 ). Five patients underwent pouch excision (ruptured aortic aneurysm 1; liver transplantation 1; recurrent disease 1; intractable pouchitis 1). The mean number of pouch evacuations per 24 hours in 71 patients with intact pouches was 8 (range 4-16) after ileostomy reversal, 7 (range 4-12) after 12 months, and 6 (range 2-13) after 75 months follow up. The most common morbidity observed was pouchitis $(37 \%)$, anastomotic stricture requiring dilatation $(13 \%)$, and frequent incontinence $(7 \%)$. Thirty-one patients $(37 \%)$ require long term use of constipating medication. These functional results are comparable to previously published outcome for IPAA in patients with CUC alone or indeterminate colitis (PB McIntyre et al, Dis Colon Rectum 1995;38;51-54).

Conclusions: there is a $20 \%$ rate of pouch failure in patients with cancer complicating CUC or FAP. Failure to benefit from an IPAA relates primarily to the need for radiotherapy or progression of disease.

\section{RECTOCELE REPAIR: A TRANSANAL APPROACH}

Kumar D. Department of Colorectal Surgery, St George's Hospital, Tooting, London, U.K.

Rectoceles represent a prolapse of the anterior rectal wall into the vagina. Since the patients present with complaints of a bulge in the vagina, the conventional approach to the repair of rectoceles is transvaginal in the form of posterior colporaphy. We have previously shown that using this approach there is a high incidence of sexual and bowel symptoms post-operatively. Thirty eight patients with a significant rectocele ( $>15 \%$ retention in the rectocele) have been treated using a transanal approach. The mean age of the patients was 46 years. All patients complained of a vaginal bulge and impaired rectal evacuation. Rectal mucosa and submucosa overlying the rectocele was dissected free of the circular muscle in the anterior half of the rectum for approximately 8 to $10 \mathrm{cms}$. The circular muscle was plicated using non-absorbable sutures longitudinally. The redundant mucosa was excised and the mucosal defect repaired with absorbable sutures. The mean follow up is $\mathbf{8}$ months (range 2 to 22 months). In 16 of the 38 patients postoperative isotope defaecography was performed to identify a residual anatomical defect. In all 16 there was no evidence of a rectocele. Symptomatically all patients improved, both in the feeling of a bulge in the vagina as well as rectal emptying. None of the patients complained of sexual problems post-operatively. These results suggest that transanal approach to the repair of rectoceles may be preferable to the conventional transvaginal repairs. 
DIABETIC DIARRHOEA AND FAECAL INCONTINENCE; VALUE OF COMBINED ENDOANAL ULTRASOUND SCAN (EAUSS) AND ANORECTAL PHYSIOLOGY STUDIES (ARP).

A. Kumar, K.J. Moriarty, M. Gowland, A. Ghandi, G. Hosker, J. Hobbiss. Depts. Gastroenterology, Radiology and Surgery, Bolton Hospitals NHS Trust, Bolton, BL4 OJR and Dept. of Urological Gynaecology, St. Mary's Hospital, Manchester, M13 0JH.

BACKGROUND AND AIM : Faecal incontinence occurs in about $20 \%$ of diabetic patients. We have investigated the aetiology of faecal incontinence in 15 diabetic patients using EAUSS and ARP.

PATIENTS : Fifteen patients ( 12 female) with a history of diabetes mellitus and faecal incontinence were studied. No patient had a history of abnormal birth trauma or prolonged second stage of labour and none had undergone ano-rectal surgery or anal stretch.

METHODS : EAUSS was performed to determine anatomical integrity. Anorectal physiology was assessed using anal manometry (resting tone and maximum voluntary squeeze pressure), assessment of rectal sensation and measurement of pudendal nerve latency. We also examined the efficacy of therapeutic intervention with Clonidine, Fybogel and Loperamide in 9 patients.

RESULTS : EAUSS was within normal limits in 13 of 15 patients. Of the other 2, there was a defect noted anteriorly in the external sphincter in one; and in another, the internal sphincter was abnormal but intact. Seven patients had either unilateral or bilateral prolonged pudendal nerve latencies. Three patients had a reduced resting anal pressure and 4 reduced voluntary squeeze pressure. Hypersensitivity of the rectum ( 3 patients) and reduced rectal sensation ( 3 patients) were also demonstrated. Eight of nine patients responded well to either Clonidine, Imodium or Fybogel or a combination of these.

CONCLUSIONS : No common aetiological factor was identified in these diabetic patients with faecal incontinence. Anatomical defects, pudendal neuropathy and abnormal rectal sensation have all been demonstrated. This series illustrates the importance of full anorectal assessment in diabetic patients with faecal incontinence, using both ano-rectal physiology studies and endo-anal ultrasonography.

QUALITY OF LIFE ASSESSMENT IN PATIENTS WITH FAECAL INCONTINENCE. S Plusa, A Sharpe, A Read, B Slater, J Varma. Department of Surgery, The Medical School, The University of Newcastle upon Tyne, NE2 4HH.

Faecal incontinence is a distressing condition which often results in a serious limitation of an individual's activity and a decrease in their perceived quality of life. This study documents the assessment of quality of life by two methods in incontinent patients and compares them to a simple incontinence score.

Fifteen patients ( 11 female) with a mean age of 57 years (range 25-86) were questioned at clinic attendance. They had a median incontinence score ${ }^{1}$ of 11 (interquartile range 10-14, 20=complete incontinence). Five were incontinent to liquid faeces alone and nine to formed stool.

Psychological health was assessed by the General Health Questionnaire $^{2}$ (GHQ) and general quality of life by the Gastrointestinal Quality of Life Index ${ }^{3}$ (GIQLI). Median GHQ score was 22 (interquartile range 13-28. 0 =best possible, worst 64 ). Median GIQLI score was 102 (interquartile range 79-119. 0=worst possible, 144=best). There was good correlation between the GHQ and GIQLI (coefficient $-0.725, p=0.002$ ), but neither the GHQ (coefficient $0.345, p=0.2$ ) nor the GIQLI (coefficient -0.316 , $\mathrm{p}=0.25$ ) correlated with the incontinence score.

The impact of faecal incontinence on quality of life is complex and is not simply correlated to the severity of the condition. A simple incontinence score is therefore inadequate as a measure of morbidity. More detailed examination is needed to allow adequate assessment of treatment and to reveal the true impact on a patient's life.

1 Jorge JMN et al. Dis Col Rect 1993;36:77-97

2 Goldberg DP et al. Psychol Med 1979;9:139-145

3 Eypasch E et al. Brit J Surg 1995;82:216-222 